\title{
Hurford's constraint, the semantics of disjunction, and the nature of alternatives
}

\author{
Ivano Ciardelli ${ }^{1}$ • Floris Roelofsen ${ }^{1}$
}

Published online: 16 March 2017

(C) The Author(s) 2017. This article is an open access publication

\begin{abstract}
This paper contributes to two recent lines of work on disjunction: on the one hand, work on so-called Hurford disjunctions, i.e., disjunctions where one disjunct entails another, and on the other hand, work in alternative and inquisitive semantics where disjunction has been argued to generate multiple propositional alternatives. We point out that Hurford effects are found not only in disjunctive statements, but also in disjunctive questions. These cases are not covered by the standard accounts of Hurford phenomena, which assume a truth-conditional treatment of disjunction. We show that inquisitive semantics facilitates a unified explanation of Hurford phenomena in statements and questions. We also argue that Hurford effects provide an empirical handle on the subtle differences between inquisitive semantics and alternative semantics, providing insight into the notion of alternatives and the notion of meaning adopted in these two frameworks.
\end{abstract}

Keywords Hurford disjunctions - Inquisitive semantics · Alternative semantics · Questions · Redundancy · Exhaustification

\section{Introduction}

Hurford disjunctions are disjunctions in which one disjunct entails another, either logically or relative to a specific context. Based on examples like those in (1) below, Hurford (1974) suggested that such disjunctions are always infelicitous, a generalization that is now referred to as Hurford's constraint.

\footnotetext{
Floris Roelofsen

f.roelofsen@uva.nl

Ivano Ciardelli

i.a.ciardelli@uva.nl

1 ILLC, Amsterdam, The Netherlands
} 
(1) a. \#John is American or Californian.

b. \#That painting is of a man or a bachelor.

c. \#The value of $x$ is different from 6 or greater than 6 .

However, Gazdar (1979) noted many apparent counterexamples to this generalization:

(2) a. Mary read most or all of the books on this shelf.

b. John and Mary have three or four kids.

c. Mary is having dinner with John, with Bill, or with both.

In subsequent work, these observations have been accounted for as follows. ${ }^{1}$ First, it has been proposed that the disjunctions in (1) are infelicitous because they involve redundancy, in the sense that the disjunction as a whole is equivalent to one of the individual disjuncts (Simons 2001; Katzir and Singh 2013; Meyer 2013, 2014). Second, the contrast between the infelicitous cases in (1) and the felicitous ones in (2) has been accounted for in terms of exhaustive strengthening. The idea is that the weak disjuncts in (2), unlike those in (1), naturally receive an exhaustive interpretation (e.g., most $\rightsquigarrow$ most but not all, while American $\Varangle \rightarrow$ American but not Californian). Under such an exhaustive interpretation, the disjunction as a whole is no longer equivalent to one of the individual disjuncts, so Hurford's constraint is satisfied (Chierchia et al. 2009, 2012).

The starting point of this paper is the observation that the patterns observed by Hurford and Gazdar obtain not only in disjunctive statements but also in disjunctive questions. For instance, the question in (3a) below is just as infelicitous as Hurford's declarative (1a), and (3b) is as felicitous as its declarative counterpart (2a).

a. \#Is John American, or Californian?

b. Did Mary read most of the books on this shelf, or all of them?

Clearly, we would hope — and expect — that these observations could be explained by the same general principles that have been proposed to explain the original observations, namely, a ban against redundant operations and the availability of exhaustive strengthening as a way of obviating such redundancy. However, whether such an explanation is possible crucially depends on the way the disjunctions in $(3 a, b)$ are analyzed. We will see that under the most widely adopted treatment of disjunction in questions, cast in the framework of alternative semantics (Hamblin 1973), the explanation does in fact not go through. By contrast, we will see that inquisitive semantics (Ciardelli et al. 2013, 2015) provides a setting in which Hurford effects in statements and questions can be explained uniformly as resulting from a ban against redundant operations combined with the availability of local exhaustification.

The semantic frameworks of alternative semantics and inquisitive semantics both refine standard truth-conditional semantics by associating sentences with sets of propositions_-called propositional alternatives — rather than with single propositions. In both frameworks, disjunction typically gives rise to multiple alternatives, each contributed by one of the disjuncts. In spite of these similarities, however, there are also subtle but fundamental differences between these frameworks in the underlying notion

\footnotetext{
1 We only give a brief summary of the main ideas here. More details will be provided in Sect. 2.
} 
of meaning and the way alternatives are construed. We will show that there is a direct connection between these differences and the distinct predictions made in the domain of Hurford disjunctions. In doing so, we hope to illustrate that the abstract logical features of a semantic framework can have very concrete empirical repercussions.

Summing up, in this paper we will argue that inquisitive semantics makes it possible to obtain a more comprehensive account of Hurford phenomena, as they occur not only in statements but also in questions. In doing so, we will also show that Hurford disjunctions provide an empirical handle on the subtle but fundamental differences between alternative and inquisitive semantics.

The paper is organized as follows. Section 2 prepares the ground by specifying a baseline theory of Hurford disjunctions in declarative sentences in terms of redundancy and exhaustive strengthening, based on Simons (2001), Katzir and Singh (2013), and Chierchia et al. (2009, 2012). Section 3 starts with the empirical observation that Hurford effects occur uniformly across declarative and interrogative sentences. Further, it shows that under the standard treatment of disjunction in questions provided by alternative semantics, the explanation of Hurford's constraint in terms of redundancy that was proposed for statements does not carry over to questions. Section 4 then shows that the treatment of disjunction in inquisitive semantics does lead to a uniform theory of Hurford effects across statements and questions. Section 5 connects the found difference between alternative and inquisitive semantics w.r.t. Hurford disjunctions to more fundamental differences in the notion of alternatives and in the structure of the semantic space adopted in these two frameworks. Finally, Sect. 6 summarizes and concludes.

\section{Background on Hurford's constraint}

\subsection{Redundancy}

Why would Hurford's sentences in (1) be infelicitous? As mentioned above, an appealing hypothesis that has been proposed in the literature is that this has to do with redundancy (Simons 2001; Katzir and Singh 2013; Meyer 2013, 2014). There are various ways to make this idea more precise. We will focus here on Katzir and Singh's (2013) proposal, which is as follows: ${ }^{2}$

\footnotetext{
2 On Katzir and Singh's account, redundancy is not checked at a global level, i.e., at the level of a full utterance, but rather more locally, i.e., each time a complex constituent is formed by applying a binary operator to two arguments. Simons' proposal is very similar to Katzir and Singh's. Meyer (2013, 2014), on the other hand, derives Hurford's constraint from a more global redundancy principle. Her proposal crucially relies on the assumption that every declarative sentence involves a covert modal operator K, which is interpreted by default relative to the speaker's epistemic state. For instance, if Bill says "John left", then this sentence is parsed as [ $\mathrm{K}_{\mathrm{Bill}} \mathrm{John}$ left] and interpreted as saying that Bill believes that John left. This assumption clearly raises several thorny issues, e.g., concerning embedding and propositional anaphora. In particular, it is not clear how the account would extend to questions, which will be our main concern in the present paper. Evidently, if Bill asks "Did John leave?", this sentence cannot be parsed as [K $\mathrm{K}_{\text {Bill }}$ whether John left]. We therefore focus on Katzir and Singh's local redundancy account.
} 
(4) Local redundancy principle (Katzir and Singh 2013)

A sentence is deviant in a context $c$ if its logical form contains a node $O(A, B)$ which is obtained by application of a binary operator $O$ to two $\operatorname{arguments} A, B$, and the outcome is semantically equivalent, relative to $c$, to one of the arguments on its own.

This principle is formulated in terms of a notion of contextual equivalence. Katzir and Singh take a context $c$ to be a set of possible worlds, namely those worlds that are compatible with the information available in $c$, and define contextual equivalence as follows ${ }^{3}$ :

\section{Contextual equivalence (Katzir and Singh 2013)}

Two sentential constituents $A$ and $B$ are equivalent relative to a context $c$ just in case $A$ and $B$ are true in exactly the same worlds in $c$.

Let us first see how the local redundancy principle predicts Hurford's constraint for sentential disjunctions. After that, we will suggest a slight amendment in order to let the principle apply to sub-sentential disjunctions as well. Recall that, in standard truth-conditional semantics, the meaning of a sentence $A$ is taken to be a proposition $|A|$, which amounts to the set of worlds where the sentence is true. A sentence $A$ entails a sentence $B$ in case $B$ is true whenever $A$ is true, i.e., in case $|A| \subseteq|B|$. Moreover, sentential disjunction is taken to yield the union of two propositions, that is, $|\operatorname{or}(A, B)|=|A| \cup|B|$.

Now, suppose that the logical form of a sentence contains a node at which disjunction applies to two sentential constituents $A$ and $B$ where $|A| \subseteq|B|$ : then, $|\operatorname{or}(A, B)|=|A| \cup|B|=|B|$. So, we have a node at which a binary operator yields an outcome which is semantically equivalent to one of the inputs. The given logical form thus breaches Katzir and Singh's local redundancy principle. In this way, Hurford's constraint is explained as a particular consequence of a more general ban against local redundancies.

Notice that this explanation relies squarely on the classical treatment of disjunction as forming the union of two propositions. In the next section, we will need to consider other treatments of disjunction, in order to make sense of occurrences of disjunction in alternative questions; crucially, we will see that not all of these treatments allow for a replication of Katzir and Singh's argument.

For simplicity, we have formulated the argument here without making reference to context. In many concrete cases, however, contextual background information plays a crucial role. To see this, consider (6) below, a version of Hurford's classic example ((1a) above) with full sentential disjuncts.

(6) \#John is American or he is Californian.

\footnotetext{
3 The account of Simons (2001) differs from that of Katzir and Singh (2013) in that it assumes a notion of contextual equivalence which is not just sensitive to the information available in the given context, but also to the current question under discussion. Since this refinement is not immediately relevant for our purposes here, we leave it out of consideration.
} 
This sentence is only infelicitous in a context in which it is known that being Californian implies being American. For someone who lacks this background knowledge, the sentence is acceptable. This is accounted for, because relative to a context where there is no background information on the relation between being Californian and being American, the disjunction John is American or he is Californian is not equivalent to any of its disjuncts, and is therefore not predicted to be infelicitous. On the other hand, relative to any context where it is known that being Californian implies being American, John is American or he is Californian clearly is equivalent to one of the disjuncts, namely to John is American. In any such context the sentence is therefore predicted to be infelicitous.

\subsection{A small amendment}

Now let us return to Hurford's original example (1a), John is American or Californian, where the disjunction applies to two non-sentential constituents, American and Californian. In this case, Katzir and Singh's principle does not immediately apply, because contextual equivalence is only defined for sentential constituents. At first sight it may seem that this problem can be overcome simply by generalizing the notion of contextual equivalence to non-sentential constituents in the obvious way. That is, we may say that two constituents of arbitrary type are equivalent relative to a context $c$ just in case they have the same extension in all worlds in $c$. In particular, the property-denoting constituents American and American or Californian would then be equivalent relative to a context $c$ just in case the extensions of the two properties coincide in every world in $c$, which is simply to say that it is known in $c$ that being Californian implies being American. This would indeed suffice to deal with Hurford's example (1a).

However, there are other cases where this generalized notion of contextual equivalence does not suffice, and it is really the local redundancy principle itself that needs to be amended. Consider the following example:

(7) \#Isabelle de Lusignan is a descendant of King William or his son.

The disjunction King William or his son is not contextually equivalent to either one of its disjuncts on any reasonable notion of contextual equivalence. However, the sentence in (7) as a whole is equivalent to one where the disjunction is replaced by just one of its disjuncts:

Isabelle de Lusignan is a descendant of King William.

What this shows is that we cannot always determine whether a non-sentential disjunction is redundant just by looking at the disjunctive phrase itself. We have to look at the sentential constituent that the phrase is part of. ${ }^{4}$ Further confirmation of this point comes from the fact that the disjunction King William or his son is perfectly felicitous when part of other sentences, like (9).

\footnotetext{
4 A reviewer notes that there are views on the syntax-semantics interface under which the logical form of (7), unlike its surface form, in fact involves sentential disjunction. Under such views, the amendment proposed here would not be necessary.
} 
(9) Isabelle de Lusignan is married to King William or his son.

These considerations lead us to the following generalized version of Katzir and Singh's local redundancy principle.

(10) Generalized local redundancy principle

A sentence is deviant in a context $c$ if its logical form contains a node $O(A, B)$ obtained by application of a binary operator $O$ to two arguments $A$ and $B$ such that, if $S$ is the smallest sentential constituent containing $O(A, B)$, then $S[O(A, B)]$ is equivalent, relative to $c$, to either $S[A]$ or $S[B]$.

\subsection{Exhaustification}

So far, we saw that a general ban against redundancy predicts the infelicity of Hurford disjunctions, thus accounting for the oddness of the sentences in (1). But is this prediction not too strong? What about Gazdar's apparent counterexamples to Hurford's constraint in (2)? How to explain the felicity of these cases?

Chierchia et al. $(2009,2012)$ show that their grammatical theory of exhaustive strengthening accounts for the contrast between Hurford's examples in (1) and Gazdar's examples in (2). According to this theory, the logical form of a sentence may contain occurrences of a covert exhaustification operator, exh, which behaves similarly to only: it strengthens the meaning of the constituent to which it applies, making it exhaustive relative to the scalar alternatives for that constituent.

Now, in Gazdar's examples, the weak disjunct naturally receives an exhaustive interpretation, under which it is no longer entailed by the other disjunct.
a. Mary read most of the books. $\rightsquigarrow$ not all
b. John and Mary have three kids. $\rightsquigarrow$ not four
c. Mary is having dinner with John. $\rightsquigarrow$ not with Bill, Sue, ...

By contrast, the weak disjuncts in Hurford's examples can not receive such a strengthened interpretation.
a. John is American. $\not \rightarrow$ not Californian
b. That painting is of a man. $\not \rightarrow$ not a bachelor
c. The value of $x$ is different from 6. $\psi \rightarrow$ not greater than 6

These observations provide the basis for an explanation of the contrast between (1) and (2). To see this, consider first a Hurford disjunction ' $A$ or $B$ ' of the felicitous kind exemplified in (2), where $A$ is the weak disjunct and $B$ the strong one. One possible logical form for the disjunction is simply $\operatorname{or}(A, B)$, which is ruled out by the local redundancy principle. However, the grammatical theory of exhaustive strengthening allows for another logical form, namely $\operatorname{or}(\operatorname{exh}(A), \operatorname{exh}(B))$, in which the disjuncts are exhaustified prior to the application of disjunction. Now, in each of the examples in (2), exh $(A)$ is incompatible with $B$, and thus, a fortiori, it is incompatible with $\operatorname{exh}(B)$. Since both $\operatorname{exh}(A)$ and $\operatorname{exh}(B)$ are consistent, this ensures that there is no entailment between $\operatorname{exh}(A)$ and $\operatorname{exh}(B)$. Thus, the logical form $\operatorname{or}(\operatorname{exh}(A), \operatorname{exh}(B))$ 
satisfies the local redundancy principle, which accounts for the fact that the sentence is perceived as felicitous. ${ }^{5}$

Now consider a Hurford disjunction ' $A$ or $B$ ' of the infelicitous kind exemplified in (1). Again, the basic logical form $\operatorname{or}(A, B)$ is incompatible with the local redundancy principle. Moreover, in this case exhaustification does not improve the situation, leaving the meanings of the disjuncts unchanged. ${ }^{6}$ As a consequence, the second logical form available for the sentence, $\operatorname{or}(\operatorname{exh}(A), \operatorname{exh}(B))$, violates the local redundancy principle as well. Thus, in this case no logical form for the sentence satisfies the redundancy principle, which explains why the sentence is perceived as infelicitous.

Notice that, besides discriminating in a principled way between felicitous and infelicitous Hurford disjunctions, the theory of Chierchia et al. (2009, 2012) also makes a particular prediction about the interpretation of felicitous Hurford disjunctions. Namely, it predicts that the only available interpretation for such a disjunction is one in which the disjuncts are interpreted exhaustively. This turns out to be correct. Consider the following example from Chierchia et al. (2009).

(13) Either John solved two exercises, or he solved all of them.

This sentence is unambiguously false if John solved exactly three out of five exercises. This demonstrates that, indeed, only the reading $\operatorname{or}(\operatorname{exh}(A), \operatorname{exh}(B))$ is available for the sentence-not the reading $\operatorname{or}(A, B)$, under which the sentence would be true if John solved three out of five exercises.

In sum, the seemingly puzzling data concerning the felicity and interpretation of Hurford disjunctions have a perspicuous explanation under the assumption that (i) there is a ban against the application of disjunction to two arguments one of which entails the other, rooted in a more general ban against redundancy, and (ii) exhaustification of the weaker disjunct can, in some cases, break the entailment and render the stronger disjunct non-redundant after all. ${ }^{7}$

\section{Hurford effects in questions}

\subsection{Empirical observations}

Work on Hurford effects has focused so far on declarative sentences. However, the same effects occur in questions as well. Alternative questions corresponding to the

\footnotetext{
5 The logical forms $\operatorname{or}(\operatorname{exh}(A), B)$ and $\operatorname{or}(A, \operatorname{exh}(B))$ also seem to be allowed by Chierchia et al. (2009). We disregard them here, but our discussion does not hinge on this in any way.

6 This may be due to the fact that the relevant items are not scalar items, or it may be due to the particular structure of the space of scalar alternatives. For instance, if the stronger alternatives to American include Californian, Texan, etc., then these alternatives fully exhaust the denotation of the predicate. It is thus impossible to conjoin the predicate with the negation of the stronger alternatives without ending up with a contradiction (cf., Fox 2007; Singh 2008).

7 The theory of Chierchia et al. $(2009,2012)$ has been extended in Fox and Spector (2015) to account for some additional empirical issues concerning Hurford disjunctions, first discussed by Singh (2008) and Gajewski and Sharvit (2012). Since these issues are orthogonal to our concerns in the present paper, we do not discuss this work in detail.
} 
infelicitous disjunctive declaratives in (1) are equally infelicitous: ${ }^{8}$
a. \#Is John American, or Californian?
b. \#Is that painting of a man, or of a bachelor?
c. \#Is the value of $x$ different from 6 , or greater than 6 ?

On the other hand, alternative questions corresponding to the acceptable disjunctive declaratives in (2) are acceptable as well:

a. Did Mary read most of the books on this shelf, or all of them?

b. Do John and Mary have three kids, or four?

c. Is Mary having dinner with John, with Bill, or with both?

Thus, the contrast exhibited by disjunctive declaratives extends to alternative questions: a Hurford-type alternative question is felicitous only if the weak disjunct may be given an exhaustive interpretation so as to break the entailment.

Moreover, we saw above that if a Hurford disjunction is felicitous, its unique interpretation is the one resulting from an exhaustive interpretation of the disjuncts. This is also true for Hurford-type alternative questions. For instance, the question in (16) presupposes that John solved either exactly two exercises or all of the exercises, and it asks which of these two possibilities holds.

Did John solve two of the exercises, or all of them?

What these data show is that Hurford's constraint, i.e., the ban against application of disjunction to two arguments one of which entails the other, concerns disjunction in general: not only when it occurs in declaratives, but also when it is used to form questions. The assumption that such a general ban is in force, together with Chierchia et al.'s grammatical theory of exhaustive strengthening, accounts for the observations in (14)-(16). ${ }^{9}$ However, the important question that remains to be addressed is whether

\footnotetext{
8 Disjunctive questions like those in (14) can be pronounced in different ways, resulting in different interpretations. The intonation intended here is one involving a prosodic phrase break after the first disjunct (indicated by a comma), rising intonation on the first disjunct, and falling intonation on the second disjunct. This intonation forces an alternative question interpretation, rather than a polar question interpretation. Hurford effects also occur in polar disjunctive questions, but this by itself is not problematic for the standard approach to Hurford's constraint described above. Hurford effects in alternative questions, as we will see, do present a challenge.

9 One may perhaps suspect that in the particular domain of questions, Hurford effects could also be explained in terms of the interaction between information asked and information presupposed. It is often assumed that a question comes with the presupposition that one of its possible answers is true (Belnap 1966, among many others). In alternative questions like those in (14), this presupposition is enough to establish one of the answers to the question. Thus, a speaker asking such a question would be presupposing enough information to actually resolve the question, which seems to be sufficient reason to regard the question as deviant. However, this explanation does not carry over to Hurford-type alternative questions with more than two disjuncts, such as (i).
}

(i) \#Is John Russian, American, or Californian?

This question is as odd as its two-disjunct counterpart, (14a). However, in this case, the presupposition of the question does not establish any particular answer. Thus, the suggested argument would not explain the infelicity of this question. 
the general redundancy principle which was taken to explain the existence of Hurford's constraint in declaratives carries over to alternative questions as well. This depends crucially on the way disjunction is taken to operate in questions.

\subsection{Traditional accounts of disjunction in questions do not derive Hurford's constraint}

The explanation of Hurford's constraint in declaratives described in Sect. 2 relies on disjunction being interpreted as the operation that yields the union of two propositions. However, this is not the role that disjunction plays in alternative questions. ${ }^{10}$ In forming an alternative question, the propositions expressed by the two disjuncts are not merged into one, but rather-it seems - they are kept apart, each contributing a separate alternative to the meaning of the question.

This idea can be implemented in various ways. Arguably the most straightforward implementation has been given in the framework of alternative semantics (Hamblin 1973). In this framework, a basic clause such as Amy sang denotes a singleton set consisting of the proposition that is classically associated with the clause: $\llbracket A \rrbracket=\{|A|\}$. Disjunction is still taken to perform union (see Alonso-Ovalle 2006), but now at the level of sets of propositions. So, for a disjunction of two basic clauses $A$ and $B$, we get:

$$
\llbracket A \text { or } B \rrbracket=\{|A|\} \cup\{|B|\}=\{|A|,|B|\}
$$

This treatment of disjunction is central to several accounts of alternative questions, such as von Stechow (1991), Roelofsen and Gool (2010), Biezma and Rawlins (2012), and Uegaki (2014).

A different but closely related implementation of the idea is found in Karttunen's (1977) theory of questions. This theory follows Montague (1973) in taking semantic composition to yield primarily the extension $\llbracket A \rrbracket_{w}$ of an expression relative to a possible world. As usual, the extension of a sentential expression is taken to be a truth value. Moreover, Karttunen assumes an operator '?' which turns such an expression into what he calls a proto-question. The semantic effect of this operator can be defined as follows:

\footnotetext{
10 At least, not according to existing accounts of such questions. While a reviewer points out to us that a theory of alternative questions based on the truth-conditional analysis of disjunction is in principle conceivable, we are not aware of such proposals in the literature. Note that any such theory must be non-compositional, since once the propositions $p$ and $q$ expressed by the disjuncts are unioned by truth-conditional disjunction to form the proposition $p \cup q$, no operator that has access only to this proposition can retrieve $p$ and $q$ separately. The reviewer suggests that this problem may be overcome by assuming that the relevant operator has access not only to the truth-conditional content of the disjunction, i.e., the proposition $p \cup q$, but also to the propositions expressed by the formal alternatives of the disjunctive phrase, which may be assumed to be $p, q$, and $p \cap q$. Note, however, that as soon as the formal alternatives of a disjunctive phrase are taken to partly determine the semantic contribution of this phrase to the meaning of the sentence that it is part of, our redundancy principle should also take the semantic impact of these formal alternatives into account. After all, if a certain disjunct does not affect the truth-conditional content of the disjunctive phrase but does affect its formal alternatives, then this disjunct cannot be seen as redundant. This, in turn, would prevent a redundancy-based account of Hurford disjunctions in questions.
} 


$$
\llbracket ? A \rrbracket_{w}= \begin{cases}\{|A|\} & \text { if } w \in|A| \\ \emptyset & \text { if } w \notin|A|\end{cases}
$$

In Karttunen's account of alternative questions, too, disjunction performs union; however, this union only takes place after the proto-question operator has applied to each disjunct. The extension of the resulting question is as follows.

$$
\llbracket ? A \text { or } ? B \rrbracket_{w}=\llbracket ? A \rrbracket_{w} \cup \llbracket ? B \rrbracket_{w}=\{p \in\{|A|,|B|\} \mid w \in p\}
$$

Thus, an alternative question receives essentially the same denotation as in Hamblinstyle theories, except that this denotation is relativized to a particular world, and propositions which are false at this world are left out.

Now let us return to our main concern, Hurford effects. Assuming either of these classical accounts of disjunction in questions, does the explanation of Hurford's constraint in terms of local redundancy carry over to questions?

Consider first the simpler approach, couched in alternative semantics. Suppose $A$ strictly entails $B$, that is, $|A| \subset|B|$. Then the meaning of the whole disjunction, $\llbracket A$ or $B \rrbracket=\{|A|,|B|\}$, contains two alternatives, and is therefore distinct from the meaning of each of the two disjuncts. Under this analysis, then, the derivation of the meaning of a question like those in (14) does not involve any redundant operation. Thus, in the absence of any further stipulations, the infelicity of such questions is not predicted.

Now consider Karttunen's approach. Clearly, the redundancy constraint cannot be imposed directly at the level of extensions, lest we predict redundancy for any declarative disjunction whatsoever. Rather, the constraint should be taken to apply at the level of intensions. But even if $A$ entails $B$, the intension predicted by Karttunen for the disjunction ?A or ?B is different from the intensions of the arguments ?A, ?B. So on this theory as well, Hurford-type alternative questions involve no redundancy, and in the absence of any further stipulations their infelicity is not predicted.

We conclude that, given traditional treatments of disjunction in questions, redundancy-based accounts of Hurford effects in statements do not straightforwardly carry over to questions. ${ }^{11}$

\section{A uniform account of Hurford effects in inquisitive semantics}

\subsection{Basic notions}

Standardly, the meaning of a sentence is identified with its truth conditions. This view on meaning is limited, however: in particular, it is not suitable to analyze questions.

\footnotetext{
11 As a reviewer suggested to us, one may try to rescue traditional theories of disjunction in questions by replacing full-fledged semantic equivalence with a notion of equivalence modulo presupposition, and then relying on the exactly-one presupposition which is usually associated with alternative questions (see, e.g., Biezma and Rawlins 2012). Notice, however, that an explanation along these lines, to the extent that it can be made to work, would not be applicable to disjunctive declaratives, which do not have an exactly-one presupposition. So this approach would lead to two different explanations for what seems to be a single phenomenon, manifested in exactly the same way in declaratives and interrogatives. By contrast, we will provide a uniform redundancy-based explanation of Hurford effects, which applies across both sentence types.
} 
In inquisitive semantics the fundamental notion of truth relative to a possible world is replaced by the notion of support relative to an information state, where information states are modeled as sets of possible worlds. Intuitively, a declarative sentence is supported by an information state if this state contains enough information to establish that the sentence is true, while a question is supported by an information state if this state contains enough information to resolve the question.

The proposition expressed by a sentence in truth-conditional semantics is modeled as a set of worlds - the set of worlds where the sentence is true. Similarly, in inquisitive semantics the proposition expressed by a sentence is modeled as a set of information states-those states that support the sentence. The alternatives associated with a sentence $A$ are those information states that minimally support $A$, that is, those states $s$ that support $A$ and cannot be weakened without losing support.

$$
\operatorname{ALT}(A)=\{s \in \llbracket A \rrbracket \mid \text { there is no } t \in \llbracket A \rrbracket \text { such that } t \supset s\}
$$

Let us illustrate the approach by means of two examples. First, consider a basic declarative sentence like Amy sang. This sentence is supported in a state $s$ if it follows from the information available in $s$ that Amy sang; formally, this means that $s$ must be included in the set $\mid$ Amy sang $\mid$ of possible worlds where Amy sang. Thus, the proposition expressed by this sentence in inquisitive semantics is the following set of information states: ${ }^{12}$

$$
\llbracket \text { Amy sang } \rrbracket=\{s|s \subseteq| \text { Amy sang } \mid\}
$$

This meaning has a unique maximal element—a unique alternative-namely the set $\mid$ Amy sang $\mid$.

$$
\operatorname{ALT}(\text { Amy sang })=\{\mid \text { Amy sang } \mid\}
$$

As a second example, consider the polar question Did Amy sing?. This question is supported in a state $s$ if $s$ contains enough information to establish whether Amy sang; this holds if it follows from the information available in $s$ that Amy sang-i.e., if $s \subseteq \mid$ Amy sang $\mid$ - or if it follows from the information in $s$ that Amy didn't sing-i.e., if $s \subseteq \mid$ Amy didn't sing $\mid$. Thus, we have:

$$
\llbracket \text { Did Amy sing? } \rrbracket=\{s|s \subseteq| \text { Amy sang } \mid \text { or } s \subseteq \mid \text { Amy didn't sing } \mid\}
$$

In this case, our meaning does not contain a unique alternative; rather, it contains two distinct alternatives, which correspond to the two ways in which the question can be minimally resolved.

$$
\operatorname{ALT}(\text { Did Amy sing? })=\{\mid \text { Amy sang }|,| \text { Amy didn't sing } \mid\}
$$

\footnotetext{
12 As we will make explicit below, the logical form we assume for the declarative sentence under consideration here is $\left[\mathrm{C}_{\mathrm{dec}}\right.$ [Amy sang]], where $\mathrm{C}_{\mathrm{dec}}$ is a clause type marker which determines the word order and affects the prosody of the sentence. Semantically, $\mathrm{C}_{\mathrm{dec}}$ has no effect when applied to a simple clause like Amy sang, but, as we will see, not when applied to a disjunctive clause like Amy sang or danced.
} 


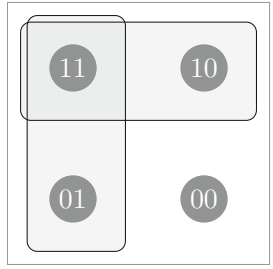

(a)

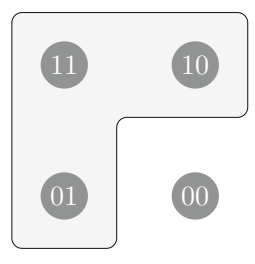

(b)

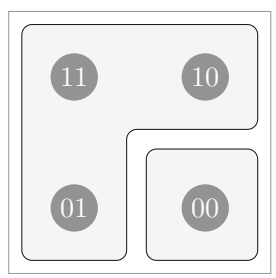

(c)

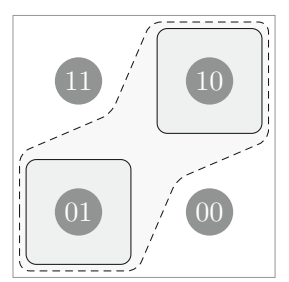

(d)

Fig. 1 A bare disjunctive phrase and three types of disjunctive sentences formed out of it. (a) [Isang or danced $\rrbracket$, (b) $\llbracket \mathrm{C}_{\mathrm{dec}}$ [sang or danced] $]$, (c) $\llbracket \mathrm{C}_{\text {pol }}$ [sang or danced] $\rrbracket$, and (d) $\llbracket \mathrm{C}_{\text {alt }}$ [sang or danced] $]$

\subsection{Inquisitive disjunction}

Just as in truth-conditional semantics and alternative semantics, disjunction is taken to perform union in inquisitive semantics as well. To see how this operation fares in the inquisitive setting, consider the disjunctive phrase Amy sang or Amy danced. The meaning assigned to this phrase is:

$$
\begin{aligned}
\llbracket \text { Amy sang or Amy danced } \rrbracket & =\llbracket \text { Amy sang } \rrbracket \cup \llbracket \text { Amy danced } \rrbracket \\
& =\{s|s \subseteq| \text { Amy sang } \mid\} \cup\{s|s \subseteq| \text { Amy danced } \mid\} \\
& =\{s|s \subseteq| \text { Amy sang } \mid \text { or } s \subseteq \mid \text { Amy danced } \mid\}
\end{aligned}
$$

This set of information states contains two alternatives, namely $\mid$ Amy sang $\mid$ and $\mid$ Amy danced $\mid$.

$$
\operatorname{ALT}(\text { Amy sang or Amy danced })=\{\mid \text { Amy sang }|,| \text { Amy danced } \mid\}
$$

The alternatives for this disjunctive phrase are depicted in Fig. 1a, where '11' is a world in which Amy sang and danced, '10' a world in which she sang but did not dance, and so on. In general, whenever disjunction applies to two simple clauses that are logically independent (i.e., neither one entails the other), the resulting meaning contains two alternatives, each of which corresponds to the proposition expressed by one of the disjuncts in standard truth-conditional semantics.

This shows that in inquisitive semantics, just as in alternative semantics, disjunction has the potential to 'collect' the alternatives associated with the two disjuncts, without collapsing them into one. This is what makes it possible to account for the role of disjunction in alternative questions.

\subsection{Hurford disjunctions are redundant in inquisitive semantics}

Let us now consider a Hurford disjunction, such as Amy danced or Amy moved, where $\mid$ Amy danced $|\subseteq|$ Amy moved $\mid$. Now any information state $s$ which is included in $\mid$ Amy danced $\mid$ must also be included in $\mid$ Amy moved $\mid$. So the information states which are included in $\mid$ Amy danced $\mid$ or in $\mid$ Amy moved $\mid$ are simply those which are included in $\mid$ Amy moved $\mid$. We thus have: 


$$
\begin{aligned}
\llbracket \text { Amy danced or Amy moved } \rrbracket & =\{s|s \subseteq| \text { Amy danced } \mid\} \cup\{s|s \subseteq| \text { Amy moved } \mid\} \\
& =\{s|s \subseteq| \text { Amy danced } \mid \text { or } s \subseteq \mid \text { Amy moved } \mid\} \\
& =\{s|s \subseteq| \text { Amy moved } \mid\} \\
& =\llbracket \text { Amy moved } \rrbracket
\end{aligned}
$$

This shows that, in inquisitive semantics, disjoining two clauses one of which entails the other is a redundant operation. As a consequence, if we combine inquisitive semantics with Katzir and Singh's redundancy principle, we predict that a logical form is always infelicitous if it contains a node at which disjunction applies to two arguments one of which entails the other. ${ }^{13}$ Thus, Hurford's constraint is derived.

\subsection{A uniform account of Hurford's constraint across disjunctive environments}

What exactly have we just shown: that Katzir and Singh's explanation of Hurford's constraint in declaratives can be replicated in inquisitive semantics, or that the inquisitive treatment of disjunction together with Katzir and Singh's redundancy principle yields an explanation for Hurford's constraint in alternative questions? The answer is: both. Let us explain why. ${ }^{14}$

We assume, with Katzir and Singh, that Hurford's constraint is to be explained in terms of a local notion of redundancy. That is, we assume that redundancy in Hurford disjunctions is detected at the level of the smallest sentential constituent containing the disjunctive phrase, regardless of whether this constituent is part of a declarative or an interrogative construction. Now, while theories such as Karttunen's distinguish two distinct entries for disjunction - a declarative and an interrogative one-in inquisitive semantics disjunction is taken to make the same semantic contribution across all the contexts in which it occurs. The difference between various kinds of disjunctive sentences arises not from a lexical ambiguity, but rather from various syntactic and intonational features, each of which makes a specific semantic contribution.

For the sake of concreteness, we will provide an explicit illustration of how the meaning of various kinds of disjunctive sentences can be derived from the core meaning that inquisitive semantics assigns to a disjunctive phrase. The details of these derivations are to a large extent immaterial for our present purposes; the important point is that the same inquisitive disjunction operator is taken to be at play in all of the relevant sentence types, and that by detecting redundancy at the level of the smallest sentential constituent containing this disjunction operator we obtain a general redundancy-based explanation of Hurford effects across declarative and interrogative constructions.

In English and many other languages, the interpretation of a disjunctive sentence depends on factors which include clause type marking (e.g., declarative/interrogative word order) and intonation (e.g., pitch contour, prosodic phrase structure). This is illustrated in (27)-(29) below, where $\uparrow$ and $\downarrow$ indicate typical rising and falling pitch

\footnotetext{
13 The same argument applies to contextual Hurford disjunctions, provided that we replace Katzir and Singh's notion of contextual equivalence with its inquisitive counterpart: $A$ and $B$ are equivalent relative to a context $c$ if and only if they are supported by exactly the same information states $s \subseteq c$.

14 Thanks to an anonymous reviewer for prompting us to address this question more explicitly.
} 
contours, respectively, and hyphenation and commas indicate the absence and presence of prosodic phrase breaks, respectively: ${ }^{15,16}$

Amy sang-or-danced $\downarrow$.

[disjunctive declarative]

Did Amy sing-or-dance $\uparrow$ ?

[disjunctive polar question]

Did Amy sing $\uparrow$, or dance $\downarrow$ ?

[alternative question]

While these three disjunctive sentences contain exactly the same lexical material, they receive different interpretations. The disjunctive declarative in (27) provides the information that Amy sang or danced, and does not raise any issue. The disjunctive polar question in (28) does not provide any information, but does raise an issue which can be resolved by establishing that Amy sang or danced, or by establishing that she didn't do either. Finally, the alternative question in (29) presupposes that Amy either sang or danced, and raises an issue which can be resolved by establishing which of the two things she did.

We will sketch a way to derive these different interpretations in inquisitive semantics, under the assumption that the sentences in (27)-(29) all contain the same disjunctive phrase, Amy sang or danced, but involve different clause type operators, which we will denote as $\mathrm{C}_{\mathrm{dec}}, \mathrm{C}_{\mathrm{pol}}$, and $\mathrm{C}_{\mathrm{alt}}$, respectively. So, syntactically (27)-(29) are represented as follows:

\section{$\left[\mathrm{C}_{\mathrm{dec}}\right.$ [Amy sang or danced]]}

[ $\mathrm{C}_{\text {pol }}[$ Amy sang or danced $\left.]\right]$

$\left[\mathrm{C}_{\mathrm{alt}}[\right.$ Amy sang or danced $\left.]\right]$

These representations are simplistic, but suffice to illustrate how the inquisitive treatment of disjunction allows us to derive the meaning of various types of disjunctive sentences in a uniform way, and which other semantic operations, besides the disjunction operator itself, may be taken to play a role in the interpretation of these sentences. While a full account of disjunctive sentences should specify how these additional semantic operations are connected to the various syntactic and intonational differences between (27)-(29), it suffices for our purpose here to assume that these operations are all 'packed into' $\mathrm{C}_{\mathrm{dec}}, \mathrm{C}_{\mathrm{pol}}$, and $\mathrm{C}_{\mathrm{alt}} \cdot{ }^{17}$

We may assume that $C_{\text {dec }}$ 'merges' the two alternatives generated by the disjunction into one big alternative, consisting of all worlds where Amy either sang or danced. Thus, the meaning of the declarative (27) is the one depicted in Fig. 1b. Similarly, we

\footnotetext{
15 A rough indication of the relevant intonation patterns suffices for our purposes here; for a more detailed description and experimental work on the intonation of disjunctive sentences see Bartels (1999) and Pruitt and Roelofsen (2013).

16 Besides disjunctive polar questions and alternative questions, exemplified in (28) and (29), there is one other type of disjunctive questions, one which has a prosodic phrase break after the first disjunct, just like alternative questions and unlike polar disjunctive questions, but rising intonation on both disjuncts, unlike alternative questions. To simplify the discussion we do not explicitly take such questions into consideration here; see, e.g., Roelofsen and Gool (2010), Pruitt and Roelofsen (2011), and Roelofsen and Farkas (2015). 17 For a less condensed version of the account we refer to Roelofsen (2013b) and Roelofsen and Farkas (2015).
} 
may assume that $\mathrm{C}_{\mathrm{pol}}$ also merges the two alternatives generated by the disjunction into one big alternative, but then adds the complement of this alternative, which consists of all worlds where Amy neither sang nor danced. Thus, the meaning of (28) is depicted in Fig. 1c. Finally, we may assume that $\mathrm{C}_{\mathrm{alt}}$ strengthens both alternatives in such a way that they become mutually exclusive (removing the overlap between them), and that it further adds a presupposition that one of these strengthened alternatives holds. Thus, the meaning of (29) is depicted in Fig. 1d, where the dashed boundary represents the presupposition of the question. ${ }^{18}$

In sum, then: we have shown that the meaning of different types of disjunctive sentences-disjunctive declaratives, disjunctive polar questions, and alternative questions - can be derived based on a uniform treatment of disjunction, i.e., without assuming that or means different things in different types of sentences. Rather, the variation in meaning results from the different clause type markers, which are connected to the variation in word order and intonation between the relevant sentence types. ${ }^{19}$

Let us stress once more the importance of this fact for our current concerns. As we saw, when the inquisitive disjunction operator is applied to two arguments one of which entails the other, the result is simply equivalent to the weaker disjunct. Thus, a disjunction in a Hurford configuration is always redundant, regardless of the particular kind of construction in which it occurs. Assuming a ban against redundant operations, this provides a uniform explanation for the general oddness of Hurford disjunctions across disjunctive declaratives and interrogatives. ${ }^{20}$

\subsection{Striking the right balance}

The ways in which truth-conditional semantics, alternative semantics, and inquisitive semantics differ in their treatment of disjunction can be illustrated pictorially as

\footnotetext{
18 This existential presupposition may be assumed to be part of any interrogative sentence, though whenever the alternatives generated by the sentence cover the entire set of possible worlds-as is the case, e.g., for the disjunctive polar question in (28) — the existential presupposition will be trivial.

19 Note that, as already anticipated in footnote 10, such a uniform and fully compositional account is not possible if we assume the standard truth-conditional treatment of disjunction, where the semantic value of a disjunctive clause is a single proposition, without any trace of what was contributed by each individual disjunct. This proposition does not contain sufficient information to derive the meaning of the corresponding alternative question in a compositional way. For this, the semantic value that $\mathrm{C}_{\text {alt }}$ takes as its input should make it possible to recognize the contribution of each individual disjunct.
}

20 Hurford's constraint is also operative in imperatives:

(i) a. \#Get an American or a Californian to do this job!

b. \#Find me a man or a bachelor!

c. \#Let the value of $x$ be different from 6 or greater than 6 .

It has been argued that, in order to account for free-choice effects, disjunction in imperatives has to be treated as generating multiple alternatives, just as in alternative questions (Aloni 2007; Aloni and Ciardelli 2013). This idea can be implemented in inquisitive semantics, which would allow us to explain Hurford effects like those in (i) exactly in the same way as Hurford effects in declaratives and interrogatives (see Ciardelli and Aloni 2016). 
follows. Suppose that $A, B$, and $C$ are basic clauses expressing the following propositions, where the outer square represent the entire logical space, and the shaded areas represent the sets $|A|,|B|$, and $|C|$, respectively.
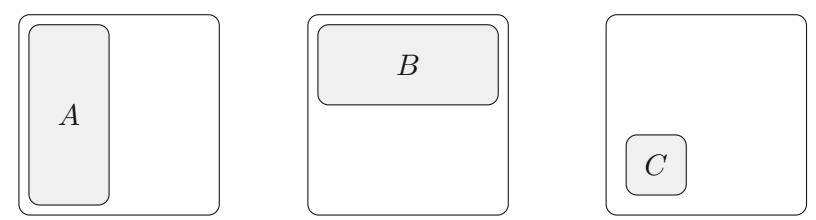

Notice that $A$ and $B$ are logically independent, while $C$ entails $A$ and is inconsistent with $B$. Now consider the non-Hurford disjunction ' $A$ or $B$ ' and the Hurford disjunction ' $A$ or $C$ '. Figure 2 represents the meaning of these disjunctions in truthconditional semantics, alternative semantics, and inquisitive semantics. In the case of truth-conditional semantics, we depict the proposition expressed, while in the case of alternative and inquisitive semantics, we depict the relevant sets of alternatives.

Truth-conditional semantics

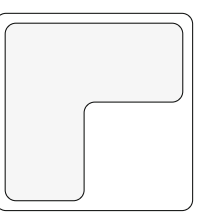

A or $C$

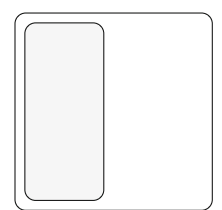

Alternative semantics
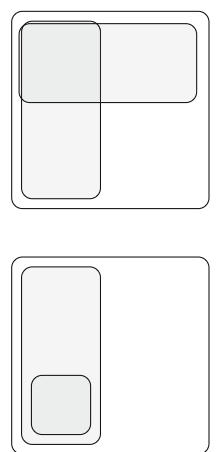

Inquisitive semantics
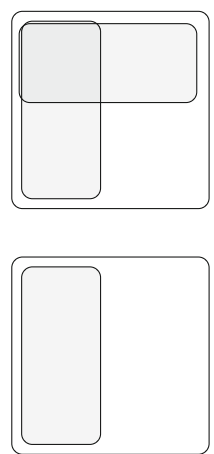

Fig. 2 ' $A$ or $B$ ' and ' $A$ or $C$ ' in truth-conditional, alternative, and inquisitive semantics 
In the case of the non-Hurford disjunction ' $A$ or $B$ ', alternative semantics and inquisitive semantics yield the same result, differing from truth-conditional semantics: the propositions expressed by the two disjuncts surface as distinct alternatives. In the case of the Hurford disjunction ' $A$ or $C$ ', however, inquisitive semantics patterns with truth-conditional semantics rather than with alternative semantics: the proposition expressed by the stronger disjunct, $C$, does not surface as a separate alternative, and the disjunction as a whole is equivalent to the weak disjunct $A$.

Thus, inquisitive semantics strikes a balance between truth-conditional semantics and alternative semantics. On the one hand, if we disjoin two logically independent sentences in inquisitive semantics, the disjunction does not conflate the propositions expressed by the two, but it keeps them apart as distinct alternatives. This feature, shared by alternative semantics but not by truth-conditional semantics, is crucial to account for the role of disjunction in alternative questions. On the other hand, if one disjunct entails the other, then the disjunction as a whole is equivalent to the weaker disjunct. This feature, shared by truth-conditional semantics but not by alternative semantics, is crucial to the explanation of Hurford's constraint in terms of redundancy. The situation is summarized in (34).

\begin{tabular}{|l|ccc|}
\hline & $\begin{array}{c}\text { Truth-conditional } \\
\text { Semantics }\end{array}$ & $\begin{array}{c}\text { Alternative } \\
\text { Semantics }\end{array}$ & $\begin{array}{c}\text { Inquisitive } \\
\text { Semantics }\end{array}$ \\
\hline $\begin{array}{l}\text { Suitable to analyze } \\
\text { alternative questions }\end{array}$ & No & Yes & Yes \\
Hurford disjunctions are redundant & Yes & No & Yes \\
\hline
\end{tabular}

\subsection{Further predictions}

\subsubsection{Contextually ruled-out disjuncts}

Combining a redundancy-based account of Hurford effects with inquisitive semantics also allows us to explain another interesting generalization: if a disjunction contains a disjunct which is inconsistent with the information available in a context $c$, then that disjunction is infelicitous in $c .^{21}$ This is true for disjunctive statements and disjunctive questions alike. As an illustration, consider (35).

(35) Context: Two friends have been following the World Cup together, and it is common knowledge among them that the two finalists are Argentina and Germany. Before the match takes place, one says to the other:

a. \#Believe me: the winner will be Argentina or Brazil.

b. \#What do you think: will the winner be Argentina, Germany, or Brazil?

Both (35a) and (35b) seem very odd. What is responsible for this?

Even though this does not seem to have been noted in previous literature, both (35a) and (35b) are in fact limit cases of contextual Hurford disjunctions. To see why,

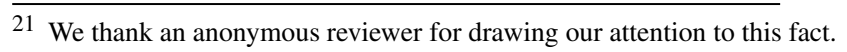


consider first the statement in (35a), and let $A$ and $B$ stand for the two disjuncts of this statement. The context $c$ described in the examples contains no worlds in which Brazil wins the World Cup; thus we have $|B| \cap c=\emptyset$. Since the empty set is a subset of any other set, we have $|B| \cap c \subseteq|A| \cap c$; that is, $B$ contextually entails $A$. Thus, (35a) is a contextual Hurford disjunction, and its oddness can be given a simple explanation: (35a) is odd because it involves a redundant disjunct.

This prediction is independent of whether we adopt a truth-conditional view on meaning or an inquisitive one. However, inquisitive semantics makes it possible to extend the same explanation to the question in (35b). In this case, too, the last disjunct contextually entails the other disjuncts and is therefore redundant. Thus, the oddness of $(35 b)$ is also explained.

\subsubsection{Conjunctions}

Hurford's constraint is a generalization about disjunctions. However, the principles that have been proposed to account for it are not specific to disjunction: they are general principles of structural economy, and as such they have repercussions for other operators as well. For instance, Katzir and Singh note that their constraint also predicts the oddness of conjunctions in which one conjunct entails the other.

a. \#Alice is 35 and she's older than 30 .

b. \#Alice is older than 30 and she is 35 .

Does inquisitive semantics provide anything new in this respect? In inquisitive semantics, one and the same conjunction operation can be applied to both statements and questions. As in standard truth-conditional semantics, this operation amounts to intersection: $\llbracket A$ and $B \rrbracket=\llbracket A \rrbracket \cap \llbracket B \rrbracket$.

When conjunction applies to statements, as in (37a), the result is essentially the same as in standard truth-conditional semantics; when it applies to questions, as in (37b), it yields a question which is resolved if and only if both conjuncts are resolved. ${ }^{22}$

a. Alice is from Wales and she is $\mathbf{3 5 .}$

b. Where is Alice from, and how old is she?

Since the behavior of conjunction when applied to statements is standard, for conjunctive statements we make exactly the same predictions as Katzir and Singh's original account. However, the move to the inquisitive setting allows us to extend these predictions to conjunctions of questions, such as the following.

a. \#Is Alice 35, and how old is she?

b. \#How old is Alice, and is she 35 ?

Consider (38a): let $\mathcal{Q}$ be the question whether Alice is 35, and $\mathcal{Q}^{\prime}$ the question how old Alice is. In order to support $\mathcal{Q}$, an information state must establish that Alice's age is 35 , or that her age is not 35 ; in order to support $\mathcal{Q}^{\prime}$, it must establish exactly what

\footnotetext{
22 For a more comprehensive discussion of the treatment of conjunction in inquisitive semantics, see, e.g., Ciardelli et al. (2015, 2016).
} 
Alice's age is. But notice that establishing Alice's age implies establishing whether this age is 35 or not. This means that any information state that supports $\mathcal{Q}^{\prime}$ also supports $\mathcal{Q}$. As a consequence, the states that support both $\mathcal{Q}$ and $\mathcal{Q}^{\prime}$ are simply those that support $\mathcal{Q}^{\prime}$. But this means that we have $\llbracket \mathcal{Q}$ and $\mathcal{Q}^{\prime} \rrbracket=\llbracket \mathcal{Q}^{\prime} \rrbracket$. Thus, (38a) contains a redundant occurrence of conjunction, which accounts for the oddness of this question. An identical story can be told to explain the infelicity of (38b). ${ }^{23}$

\section{Hurford's constraint as a window onto semantic structure}

In the previous section we have shown that Katzir and Singh's redundancy-based explanation of Hurford's constraint can be extended to questions. In doing so we encountered a difference between inquisitive semantics and alternative semantics. The former facilitates the desired extension in a straightforward way, while the latter doesn't. In this section, we investigate the source of this discrepancy, connecting it to more fundamental features of the two frameworks.

\subsection{Hurford's constraint and the nature of alternatives}

While both alternative semantics and inquisitive semantics associate sentences with sets of propositional alternatives, the notion of alternatives is conceptually quite different in the two cases.

In alternative semantics, the notion of a propositional alternative is a primitive notion and there are no constraints on which kinds of sets of propositions count as proper sets of alternatives. In inquisitive semantics, on the other hand, alternatives are defined in terms of the more basic notion of support: the alternatives associated with a sentence are those propositions that support the sentence in a minimal way. This characterization implies that sets of alternatives have to be of a particular form: two alternatives are always logically independent, that is, one is never contained in the other.

Let us refer to sets of propositions whose elements are pairwise logically independent as non-nested sets. In inquisitive semantics, then, unlike in alternative semantics, only non-nested sets of propositions are regarded as proper sets of alternatives. Since this constraint does not arise in alternative semantics, that framework has greater expressive power-it makes more meanings available. At first sight, this seems attrac-

\footnotetext{
23 There are two observations about conjunction that neither Katzir and Singh's original proposal nor its inquisitive extension directly account for. First, there are cases where the order of the conjuncts seems to matter (note that in (36) and (38) we saw cases where order does not seem to matter). The following example is from Schlenker (2008):
}

(i) $\quad$ \#John resides in Paris and lives in France.

b. John lives in France and resides in Paris.

Second, Mayr and Romoli (2016) draw attention to cases with nested conjunctions like (ii).

(ii) \#Mary is beautiful and married, and she is pregnant and married.

We refer to Katzir and Singh (2013) and Mayr and Romoli (2016) for further discussion. 
tive. However, we have seen that these additional meanings, i.e., nested sets of alternatives, are impossible to express in natural languages like English. In principle, a Hurford disjunction would be exactly the right kind of construction to express a nested set of alternatives. But such disjunctions are felicitous only if they can be re-interpreted in such a way that their sets of alternatives actually becomes non-nested, and, if such re-interpretation is possible, the resulting non-nested set constitutes the only available interpretation for the disjunction.

This seems to indicate that there is something wrong with nested sets of alternatives as meanings: the sentences that are supposed to express such sets are either infelicitous or re-interpreted as expressing non-nested sets of alternatives. From the perspective of alternative semantics this is puzzling, since nested sets of alternatives are in principle just as good as non-nested sets. Of course, it would be possible to stipulate that they are not, but since this does not follow from the way in which alternatives are construed in the framework, a stipulation of this kind would essentially amount to a semantic reformulation of Hurford's constraint, rather than an explanation thereof.

In inquisitive semantics, the puzzle does not arise, because nested sets of alternatives simply do not exist. Importantly, such sets are not ruled out by stipulation: rather, their absence immediately follows from the way in which alternatives are construed in this framework. This means that from the perspective of inquisitive semantics, what is special about Hurford disjunctions is not that they express some distinguished class of meanings, but rather that they involve redundant disjuncts, which fail to contribute an alternative to the meaning of the disjunction. As we discussed, this is precisely what makes it possible to explain the need for re-interpretation and, in case this is not possible, the infelicity of such disjunctions.

\subsection{Hurford's constraint and semantic structure}

Just as in truth-conditional semantics, the most fundamental logical relation between sentences in inquisitive semantics is entailment. Moreover, just as in truth-conditional semantics, entailment in inquisitive semantics still amounts to meaning inclusion: $A \models B \Longleftrightarrow \llbracket A \rrbracket \subseteq \llbracket B \rrbracket$. Finally, again just as in truth-conditional semantics, the space of all meanings in inquisitive semantics ordered by entailment forms a lattice. That is, every two meanings $\llbracket A \rrbracket$ and $\llbracket B \rrbracket$ have a greatest lower bound (meet) with respect to entailment, namely their intersection $\llbracket A \rrbracket \cap \llbracket B \rrbracket$, and a least upper bound (join), namely their union $\llbracket A \rrbracket \cup \llbracket B \rrbracket .{ }^{24}$ In truth-conditional semantics, this structural feature of the space of meanings is crucially exploited in the treatment of conjunction and disjunction. Namely, a conjunction ' $A$ and $B$ ' is taken to express the meet of $\llbracket A \rrbracket$ and $\llbracket B \rrbracket$, and a disjunction ' $A$ or $B$ ' is taken to express the join of $\llbracket A \rrbracket$ and $\llbracket B \rrbracket$. Since the space of meanings in inquisitive semantics still forms a lattice, precisely the same treatment of conjunction and disjunction can be given in this setting. So, while inquisitive semantics enriches the truth-conditional semantic framework, it retains its

\footnotetext{
24 The greatest lower bound of two meanings with respect to entailment is the weakest meaning that entails both, and their least upper bound is the strongest meaning that is entailed by both. For more elaborate discussion of these notions in the context of inquisitive semantics, see Roelofsen (2013a).
} 
fundamental structural features, and therefore also allows us to preserve the essence of the truth-conditional treatment of conjunction and disjunction.

Now, as discussed in detail in Roelofsen (2013a) and Ciardelli et al. (2016), this is not so for alternative semantics, where the standard notion of entailment as inclusion does not give the right results and has not been replaced by a suitable alternative notion. As a consequence, the principled treatment of conjunction and disjunction as expressing meet and join operations with respect to entailment is lost. While disjunction is still taken to yield the union of the alternative sets associated with the two disjuncts, this operation no longer has the same status and the same logical properties as in truthconditional and in inquisitive semantics. In the case of conjunction, the situation is even more problematic-taking conjunction to express intersection leads to undesirable results even for the most basic cases (see Ciardelli et al. 2016 for concrete examples).

These considerations are of a more abstract nature than the ones that we have been concerned with above. Upon closer examination, however, they bring out precisely those structural features of inquisitive semantics that are responsible for its success in accounting for Hurford's constraint in terms of redundancy. Let us see why.

Consider an arbitrary space of meanings $\mathcal{M}$ ordered by a suitable relation of semantic strength $\leq$. Suppose the space $\langle\mathcal{M}, \leq\rangle$ is a lattice; that is, suppose any two meanings $M, M^{\prime} \in \mathcal{M}$ have a meet and a join, which we will denote by $M \wedge M^{\prime}$ and $M \vee M^{\prime}$ respectively. If our semantics is based on such a space of meanings, then we can say that a sentence $A$ entails a sentence $B$ just in case the meaning of $A$ is at least as strong as the meaning of $B$ :

$$
A=B \Longleftrightarrow \llbracket A \rrbracket \leq \llbracket B \rrbracket
$$

Moreover, we can associate conjunction and disjunction with the two lattice operations:

$$
\begin{aligned}
& \text { a. } \llbracket \text { and } \rrbracket=\lambda M \cdot \lambda M^{\prime} . M \wedge M^{\prime} \\
& \text { b. } \llbracket \text { or } \rrbracket=\lambda M \cdot \lambda M^{\prime} . M \vee M^{\prime}
\end{aligned}
$$

Now, it follows from the very definition of the join operation that if $M \leq M^{\prime}$, then $M \vee M^{\prime}=M^{\prime}$. Thus, if our semantics is based on a space of meanings that constitutes a lattice, and if disjunction is taken to express the join operation in this space, this is sufficient to predict redundancy in Hurford configurations: ${ }^{25}$

$$
\text { if } A \models B \text {, then } \llbracket A \text { or } B \rrbracket=\llbracket B \rrbracket
$$

Both truth-conditional semantics and inquisitive semantics instantiate this general scheme: in both cases, semantic strength is captured by inclusion, and the join operation amounts to set-theoretic union. Thus, in truth-conditional and inquisitive semantics, Hurford disjunctions are redundant for the same fundamental reason: they involve a join operation on two arguments one of which entails the other.

This does not hold for alternative semantics. As we saw, it is not quite clear how meanings should be compared in this setting, i.e., how they should be ordered in terms

\footnotetext{
25 Likewise, if conjunction is taken to express the meet operation, redundancy is automatically predicted when one conjunct is stronger than the other. As a consequence, the infelicity of such cases can also be explained in terms of redundancy in any lattice-based semantics.
} 
of semantic strength. But suppose we manage to define a suitable ordering $\leq$. For basic clauses, whose meaning is a singleton set of propositions, we would want entailment to reduce to the classical notion; that is, we want to have that $\{|A|\} \leq\{|B|\}$ just in case $|A| \subseteq|B|$. But then, alternative semantics disjunction cannot be a join operation. For, suppose that $|A| \subset|B|$. Then the least upper bound of $\{|A|\}$ and $\{|B|\}$ would be $\{|B|\}$, but alternative semantics disjunction yields $\{|A|\} \cup\{|B|\}=\{|A|,|B|\} \neq\{|B|\}$. Thus, the fact that alternative semantics does not predict redundancy in Hurford disjunctions is directly connected to the fact that the account of disjunction as a join operation is not preserved.

\section{Conclusion}

We hope to have achieved two things in this paper. First, we hope to have demonstrated the relevance of inquisitive semantics for the theory of Hurford disjunctions: a general account of Hurford effects, both in statements and in questions, becomes available if we combine a ban against redundant operations, as proposed by Katzir and Singh (2013), with an inquisitive account of disjunction-a result that seems difficult to obtain based on other existing accounts of disjunction. Of particular importance here is that, in its treatment of disjunction, inquisitive semantics strikes a subtle balance between truth-conditional semantics and alternative semantics.

Second, we hope to have shown how the different predictions made by inquisitive semantics and alternative semantics in the domain of Hurford disjunctions are connected to some more abstract and more fundamental differences between the two frameworks. One of these differences concerns the notion of propositional alternatives: in alternative semantics this is a primitive, unconstrained notion; in inquisitive semantics, by contrast, alternatives are characterized in terms of the more basic semantic notion of support, and it follows from this characterization that sets of alternatives must have a certain form-namely, one alternative can never be nested in another. The other difference that we discussed concerns the structure of the underlying semantic space: the essential structural features of the semantic space assumed in truth-conditional semantics are preserved in inquisitive semantics, but not in alternative semantics. We argued that both of these more abstract differences between the two frameworks are directly connected to the different predictions that they yield about Hurford disjunctions. Thus, besides contributing to the theory of Hurford disjunctions as such, we hope to have illustrated that more abstract features of a semantic framework are not just important from a logical and philosophical point of view, but can also be crucial to its empirical success.

Acknowledgements We are grateful to Jeroen Groenendijk and Matthijs Westera for many discussions closely related to the ideas presented in this paper, and to two anonymous reviewers for their constructive feedback. We also gratefully acknowledge financial support from the Netherlands Organization for Scientific Research (NWO) and the European Research Council (ERC, Grant Agreement No. 680220).

Open Access This article is distributed under the terms of the Creative Commons Attribution 4.0 International License (http://creativecommons.org/licenses/by/4.0/), which permits unrestricted use, distribution, and reproduction in any medium, provided you give appropriate credit to the original author(s) and the source, provide a link to the Creative Commons license, and indicate if changes were made. 


\section{References}

Aloni, M. 2007. Free choice, modals and imperatives. Natural Language Semantics 15 (1): $65-94$.

Aloni, M., and I. Ciardelli. 2013. A logical account of free-choice imperatives. In The dynamic, inquisitive, and visionary life of $\varphi, ? \varphi$, and $\diamond \varphi$ : A festschrift for Jeroen Groenendijk, Martin Stokhof, and Frank Veltman, ed. M. Aloni, M. Franke, and F. Roelofsen, 1-17. Amsterdam: ILLC Publications.

Alonso-Ovalle, L. 2006. Disjunction in alternative semantics. Ph.D. thesis, University of Massachusetts, Amherst.

Bartels, C. 1999. The intonation of English statements and questions: A compositional interpretation. New York, NY: Routledge.

Belnap, N. 1966. Questions, answers, and presuppositions. The Journal of Philosophy 63 (20): 609-611.

Biezma, M., and K. Rawlins. 2012. Responding to alternative and polar questions. Linguistics and Philosophy 35 (5): 361-406.

Chierchia, G., D. Fox, and B. Spector. 2009. Hurford's constraint and the theory of scalar implicatures: Evidence for embedded implicatures. In Presuppositions and implicatures, ed. P. Egré, and G. Magri, 47-62. Cambridge, MA: MIT Press.

Chierchia, G., D. Fox, and B. Spector. 2012. Scalar implicature as a grammatical phenomenon. In Semantics: An international handbook of natural language meaning, vol. 3, ed. P. Portner, C. Maienborn, and K. von Heusinger, 2297-2331. Berlin: Mouton de Gruyter.

Ciardelli, I., and M. Aloni. 2016. Choice-offering imperatives in inquisitive and truth-maker semantics. Presented at 'Imperatives: worlds and beyond', Hamburg University, June 2016.

Ciardelli, I., J. Groenendijk, and F. Roelofsen. 2013. Inquisitive semantics: A new notion of meaning. Language and Linguistics Compass 7 (9): 459-476.

Ciardelli, I., J. Groenendijk, and F. Roelofsen. 2015. Inquisitive semantics. European Summer School in Logic, Language and Information lecture notes. www.illc.uva.nl/inquisitivesemantics.

Ciardelli, I., F. Roelofsen, and N. Theiler. 2016. Composing alternatives. Linguistics and Philosophy. doi:10. 1007/s10988-016-9195-2.

Fox, D. 2007. Free choice disjunction and the theory of scalar implicatures. In Presupposition and implicature in compositional semantics, ed. U. Sauerland and P. Stateva, 71-120. Basingstoke: Palgrave Macmillan.

Fox, D., and B. Spector. 2015. Economy and embedded exhaustification. MIT and Institut Jean Nicod: Manuscript.

Gajewski, J., and Y. Sharvit. 2012. In defense of the grammatical approach to local implicatures. Natural Language Semantics 20 (1): 31-57.

Gazdar, G. 1979. Pragmatics: Implicature, presupposition, and logical form. New York, NY: Academic Press.

Hamblin, C.L. 1973. Questions in Montague English. Foundations of Language 10 (1): 41-53.

Hurford, J. 1974. Exclusive or inclusive disjunction. Foundations of Language 11 (3): 409-411.

Karttunen, L. 1977. Syntax and semantics of questions. Linguistics and Philosophy 1: 3-44.

Katzir, R., and R. Singh. 2013. Hurford disjunctions: Embedded exhaustification and structural economy. In Proceedings of Sinn und Bedeutung 18, ed. U. Etxeberria, A. Fălăuş, A. Irurtzun, and B. Leferman, 201-216.

Mayr, C., and J. Romoli. 2016. A puzzle for theories of redundancy: Exhaustification, incrementality, and the notion of local context. Semantics and Pragmatics 9 (7): 1-48.

Meyer, M.-C. 2013. Ignorance and grammar. Ph.D. thesis, MIT.

Meyer, M.-C. 2014. Deriving Hurford's constraint. In Proceedings of Semantics and Linguistic Theory 24, ed. T. Snider, S. D'Antonio, and M. Weigand, 577-596. LSA and CLC Publications.

Montague, R. 1973. The proper treatment of quantification in ordinary English. In Approaches to natural language, ed. J. Hintikka, J. Moravcsik, and P. Suppes, 221-242. Dordrecht: Reidel.

Pruitt, K., and F. Roelofsen. 2011. Disjunctive questions: Prosody, syntax, and semantics. Available via www.illc.uva.nl/inquisitivesemantics: Presented at a seminar at the Georg August Universität Göttingen.

Pruitt, K., and F. Roelofsen. 2013. The interpretation of prosody in disjunctive questions. Linguistic Inquiry 44: 632-650.

Roelofsen, F. 2013a. Algebraic foundations for the semantic treatment of inquisitive content. Synthese 190 (1): 79-102. 
Roelofsen, F. 2013b. An inquisitive perspective on meaning: The case of disjunction. Stanford Linguistics Colloquium, February 2013.

Roelofsen, F., and D.F. Farkas. 2015. Polarity particle responses as a window onto the interpretation of questions and assertions. Language 91 (2): 359-414.

Roelofsen, F., and S. van Gool. 2010. Disjunctive questions, intonation, and highlighting. In Logic, language, and meaning: Selected papers from the Seventeenth Amsterdam Colloquium, ed. M. Aloni, $\mathrm{H}$. Bastiaanse, T. de Jager, and K. Schulz, 384-394. Berlin: Springer.

Schlenker, P. 2008. Be articulate: A pragmatic theory of presupposition projection. Theoretical Linguistics 34 (3): 157-212.

Simons, M. 2001. Disjunction and alternativeness. Linguistics and Philosophy 24 (5): 597-619.

Singh, R. 2008. On the interpretation of disjunction: Asymmetric, incremental, and eager for inconsistency. Linguistics and Philosophy 31 (2): 245-260.

von Stechow, A. 1991. Focusing and backgrounding operators. In Discourse particles: Descriptive and theoretical investigations on the logical, syntactic and pragmatic properties of discourse particles in German, ed. W. Abraham, 37-84. Amsterdam: John Benjamins.

Uegaki, W. 2014. Japanese alternative questions are disjunctions of polar questions. In Proceedings of Semantics and Linguistic Theory 24, ed. T. Snider, S. D'Antonio, and M. Weigand, 42-62. LSA and CLC Publications. 